Research Article

\title{
Contribution of OqxAB Efflux Pump in Selection of Fluoroquinolone-Resistant Klebsiella pneumoniae
}

\author{
Orsolya Szabo, ${ }^{1}$ Bela Kocsis $\mathbb{D}^{1},{ }^{1}$ Nikolett Szabo, ${ }^{1}$ Katalin Kristof, ${ }^{2}$ and Dora Szabo ${ }^{1}$ \\ ${ }^{1}$ Institute of Medical Microbiology, Semmelweis University, Budapest 1082, Hungary \\ ${ }^{2}$ Institute of Laboratory Medicine, Clinical Microbiology Laboratory, Semmelweis University, Budapest 1089, Hungary \\ Correspondence should be addressed to Bela Kocsis; kocsis.bela@med.semmelweis-univ.hu
}

Received 4 April 2018; Accepted 7 August 2018; Published 23 September 2018

Academic Editor: Paul-Louis Woerther

Copyright (C) 2018 Orsolya Szabo et al. This is an open access article distributed under the Creative Commons Attribution License, which permits unrestricted use, distribution, and reproduction in any medium, provided the original work is properly cited.

The role of OqxAB efflux pump in Klebsiella pneumoniae was investigated in correlation with ciprofloxacin exposure. K. pneumoniae SE23 and K. pneumoniae SE191 were isolated from urinary tract infections and were analyzed in this study. Each carried oqxAB resistance determinant and exhibited ciprofloxacin MIC of 0.06 and $0.5 \mathrm{mg} / \mathrm{L}$, respectively. Tested strains were initially exposed to their ciprofloxacin MIC values for 24 hours. Later on, the ciprofloxacin exposition has been increased to a daily $1,2,4$, and to a final $8 \mathrm{mg} / \mathrm{L}$. Total cellular RNA was extracted at 30, 60, 90, and 120 minutes of initial exposure and after every 24 hours. Quantitative reverse-transcriptase PCR was performed from each RNA sample. Mutation in gyrA and parC genes was analyzed in each strain and multilocus sequence typing (MLST) was performed. Ciprofloxacin exposure selected resistant strain from K. pneumoniae SE191; by contrast, K. pneumoniae SE23 was not adjustable to the increasing ciprofloxacin concentrations. During initial exposure, both $o q x A$ and $o q x B$ expression remained low $\left(2^{-\Delta \mathrm{Ct}}=1-2.03\right)$. However, increasing ciprofloxacin promoted $o q x B$ expression as it reached fold increase of $15.8-22.8$, while oq $x A$ expression was maintained $\left(2^{-\Delta \mathrm{Ct}}=2-2.15\right)$. An amino acid substitution Ser83Tyr in gyrA was detected in K. pneumoniae SE191, but no additional mutations occurred as consequence to ciprofloxacin exposure. MLST identified K. pneumoniae SE191 as ST274, while K. pneumoniae SE23 belonged to the novel ST2567. Ciprofloxacin concentration-dependent upregulation of oqxAB efflux pump in K. pneumoniae is clonally related and contributes to selection for higher level of fluoroquinolone resistance.

\section{Introduction}

Klebsiella pneumoniae is one of the most widespread nosocomial pathogen and responsible for various infectious diseases, including urinary tract, bloodstream, and respiratory tract infections. It is capable of developing resistance to cephalosporins, carbapenems, fluoroquinolones, aminoglycosides, and recently to polymyxins [1-3]. Fluoroquinolone resistance in $K$. pneumoniae is explained by mutations in gyrase and topoisomerase IV enzymes; however, reduced susceptibility to fluoroquinolones and lowlevel resistance is maintained by plasmid-mediated quinolone resistance (PMQR) determinants, namely, Qnr determinants, QepA and OqxAB efflux pumps, and aminoglycoside-acetyltransferase Ib-cr enzyme [4].

OqxAB efflux pump belongs to the resistance nodulation division family and it is constructed by two main domains, namely, OqxA, a periplasmic part, and OqxB, a transmembrane protein. Its efflux pump activity is described in a TolC-independent way; however, presence of an outer membrane protein enhances its effect [5]. OqxAB has been commonly found chromosomally in K. pneumoniae and usually plasmid located in other Enterobacteriaceae species. This resistance determinant is responsible to develop reduced susceptibility and even resistance to olaquindox and other fluoroquinolone agents such as ciprofloxacin, norfloxacin, and flumequine $[5,6]$. High prevalence of OqxAB has been described in K. pneumoniae on both chromosome and plasmids representing a potential reservoir of this resistance determinant [7-10]. In K. pneumoniae, upregulation of oq $x A B$ efflux pump is $\operatorname{rar} A$ mediated; by contrast, oqxR is capable of downregulating it [11-13]. It has been recently reported that AcrAB associated with overexpression of OqxAB is required for high virulence potential in K. pneumoniae [14]. 
Internationally successful high-risk clones of $K$. pneumoniae have disseminated in Hungary. These clones were fluoroquinolone resistant and harboured extendedspectrum beta-lactamases $\left(b l a_{\mathrm{CTX}-\mathrm{M}-15}\right)$ or carbapenemases $\left(b l a_{\mathrm{VIM}-4}, b l a_{\mathrm{KPC}}\right)$; however, they retained fitness and these combined features contributed to the widespread dissemination of ST11, ST15, ST147, and ST258 in hospital settings and made them responsible for the majority of healthcareassociated infections [15-17]. Recently, ST274 has emerged in adult and newborn hospital wards and has been reported in several nosocomial infections [17]. The aim of this study was to analyze role of $o q x A B$ efflux pump in selection of fluoroquinolone resistance in different $K$. pneumoniae clones.

\section{Materials and Methods}

2.1. Bacterial Strains. Two $K$. pneumoniae strains ( $K$. pneumoniae SE23 and K. pneumoniae SE191) have been included in this study. Both have been previously found positive to oq $x A B$ resistance determinant by PCR and nucleic acid sequencing and lacked all other PMQRs. Antimicrobial susceptibility testing was performed by microdilution method based on documents of the European Committee on Antimicrobial Susceptibility Testing (EUCAST), and both K. pneumoniae SE23 and K. pneumoniae SE191 were found susceptible to ciprofloxacin with MIC 0.06 and $0.5 \mathrm{mg} / \mathrm{L}$, respectively, based on EUCAST breakpoints issued in the year 2016. However, ciprofloxacin resistance breakpoint of Enterobacteriaceae has been revised by EUCAST in 2017, indicating $0.5 \mathrm{mg} / \mathrm{L}$ value as nonsusceptible to fluoroquinolones.

2.2. Exposure to Ciprofloxacin. Each tested strain has been prepared in 0.5 McFarland density in Mueller-Hinton broth and has been initially exposed to their ciprofloxacin MIC values of 0.06 and $0.5 \mathrm{mg} / \mathrm{L}$ for 24 hours at $37^{\circ} \mathrm{C}$. RNA extraction has been carried out at 30,60, 90, and 120 minutes and at 24 hours of ciprofloxacin exposition. Later on, after the initial incubation, each tested strain has been adjusted to $0.5 \mathrm{McF}$ arland density and has been exposed to an increased ciprofloxacin concentration. In the case of K. pneumoniae SE23, the peak ciprofloxacin exposition was $0.5 \mathrm{mg} / \mathrm{L}$. On the other hand, K. pneumoniae SE191 was capable of adapting to a daily increasing $1,2,4$, and $8 \mathrm{mg} / \mathrm{L}$ ciprofloxacin exposition, where each incubation at $37^{\circ} \mathrm{C}$ lasted for 24 hours. After each daily exposure, RNA extraction has been performed from each sample to analyze expression of oq $x A B$ efflux pump.

2.3. Quantitative Reverse-Transcriptase PCR Analysis for Expression of oq $x A B$. Total cellular RNA was extracted from both tested strains at given time point of incubation by RNeasy Mini Kit (Qiagen GmbH, Hilden, Germany) according to the manufacturer's instructions. The quantitative RT-PCR was carried out in a Step One Real-Time PCR System (Applied Biosystems, Thermo Fisher Scientific) with following thermal profile: $60^{\circ} \mathrm{C}$ for $30 \mathrm{sec} ; 50^{\circ} \mathrm{C}$ for $5 \mathrm{~min}$ and $95^{\circ} \mathrm{C}$ for $10 \mathrm{~min}$; 40 cycles of $95^{\circ} \mathrm{C}$ for $15 \mathrm{sec}$ and $60^{\circ} \mathrm{C}$ for $1 \mathrm{~min}$; and $60^{\circ} \mathrm{C}$ for $30 \mathrm{sec}$.

To test separate expression of the oqxA and oq $x B$ gene, the internal fragments were used to design set of primers and 6-FAM- or VIC-labelled probes. Chromosomal rpoB was chosen as housekeeping gene. All oligonucleotide primers and probes for quantitative RT-PCR were designed by Primer Express 3.0 software and are listed in Table 1. Each sample was tested in triplicate. The Ct values of genes of interest were normalized $(\Delta \mathrm{Ct})$ to the $\mathrm{Ct}$ values of housekeeping gene $r p o B$, and the relative expression of each gene of interest was calculated as $2^{-\Delta \mathrm{Ct}}$.

2.4. Analysis of Mutations in gyrA and parC Genes. Mutations in gyrA and parC genes have been investigated by PCR and nucleic acid sequencing. Set of primers was designed by online tools of MWG Eurofins Operon and synthetised by IDT Bioscience. PCR thermal profile was as follows: initial denaturation at $95^{\circ} \mathrm{C}$ for $3 \mathrm{~min}, 30$ cycles of $95^{\circ} \mathrm{C}$ for $1 \mathrm{~min}, 52^{\circ} \mathrm{C}$ for $1 \mathrm{~min}, 72^{\circ} \mathrm{C}$ for $1 \mathrm{~min}$, and an additional extension at $72^{\circ} \mathrm{C}$ for $5 \mathrm{~min}$.

2.5. Multilocus Sequence Typing (MLST) of K. pneumoniae. Each tested strain has been analyzed by multilocus sequence typing based on sequences of seven housekeeping genes, namely, gapA, infB, $m d h$, pgi, phoE, rpoB, and tonB. Oligonucleotide primers and PCR thermal profiles were applied after Diancourt et al. article [18] and as it is highlighted on MLST database website http://bigsdb.pasteur. fr/klebsiella/primers_used.html.

Amplicons of MLST, gyrA and parC PCR were analyzed by electrophoresis in a $1.5 \%$ agarose gel (Sigma-Aldrich) at $120 \mathrm{~V}$ for $20 \mathrm{~min}$ in 1xTAE (40 mM Tris- $\mathrm{HCl}$ (pH 8.3), $2 \mathrm{mM}$ acetate, and $1 \mathrm{mM}$ EDTA), and gel was stained with $0.05 \mathrm{mg} / \mathrm{L}$ GelRed dye (Biotium) and visualised on a UV transilluminator. PCR-positive amplicons were purified by QIAquick PCR Purification Kit (Qiagen GmbH, Hilden, Germany), and nucleic acid sequencing was performed by BIOMI Kft. Gödöllö, Hungary.

2.6. Statistical Analysis. Expression level differences were analyzed by $t$-test for 2 independent means.

\section{Results}

K. pneumoniae SE23 and K. pneumoniae SE191 strains were positive to $o q x A B$ by PCR and were both initially exposed to ciprofloxacin concentration of their MIC values 0.06 and $0.5 \mathrm{mg} / \mathrm{L}$, respectively. However, selection of resistant strains was successful only by K. pneumoniae SE191 and it was found to be adjustable for further ciprofloxacin exposition. Hence, multiple conditions K. pneumoniae SE23 was not capable of adapting to increased ciprofloxacin concentrations. The highest ciprofloxacin value was $0.5 \mathrm{mg} / \mathrm{L}$, where RNA could have been extracted from K. pneumoniae SE23, but no further exposition was possible. 
TABLE 1: Oligonucleotide primers and probes of this study.

\begin{tabular}{lrc}
\hline Primer or probe & Sequence & Reference \\
\hline gyrA fwd & CAGCCCTTCAATGCTGATG & Designed in the study \\
gyrA rev & CGCTTTTACTCCTTTCTGTTC & Designed in the study \\
parC fwd & CTCAATCAGCGTAATCGCC & Designed in the study \\
parC rev & AATCCTCAGCCGATCTCAC & Designed in the study \\
Kpn.rpoBF1 fwd & GTCGCGGCTGAACAAGCT & Designed in the study \\
Kpn.rpoBR1 rev & AACGGCCACTTCGTAGAAGATC & Designed in the study \\
Kpn.rpoBP1-VIC probe & CTACGGCAGGTAACC & Designed in the study \\
oqxAF1 fwd & GTCGACGGCTTACAAAAAGTGTT & Designed in the study \\
oqxAR1 rev & GCAACGGTTTTGGCGTTAA & Designed in the study \\
oqxAP1-FAM probe & ATGCCGGGTATGCC & Designed in the study \\
oqxBF1 fwd & CTGGATTTTCCGTCCGTTTAAC & Designed in the study \\
oqxBR1 rev & TTGCCTACCAGTCCCTGATAGC & Designed in the study \\
oqxBP1-FAM probe & CTGCGCAGCTCGAA & Designed in the study \\
\hline
\end{tabular}

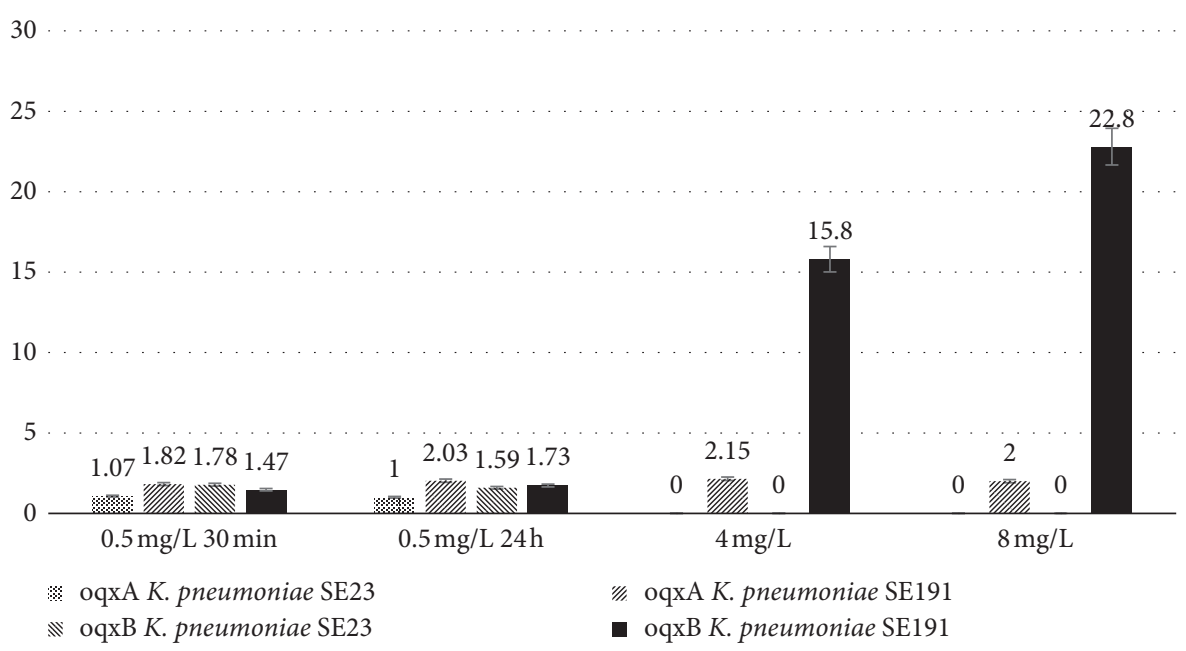

FIgURE 1: Expression in SE23 and SE191.

Quantitative RT-PCR was performed on RNA samples taken at given time points of ciprofloxacin exposure to investigate expression level differences of oq $x A B$ efflux pump. Expression in SE191 to $0.5 \mathrm{mg} / \mathrm{L}$ for 30 minutes was 1.82 and no relevant expression change to 4 and $8 \mathrm{mg} / \mathrm{L}$ as $2^{-\Delta \mathrm{Ct}}$ values were 2.15 and 2.00 . In the case of SE23 expression, $2^{-\Delta \mathrm{Ct}}$ values were 1.07 and 1 at maximum $0.5 \mathrm{mg} / \mathrm{L}$ (Figure 1).

In the case of OqxAB, expression level values calculated as $2^{-\Delta C T}$ were 1.47 and 1.73 for SE191 while in SE23 values were 1.78 and 1.59. The change in expression of SE191 to 4 and $8 \mathrm{mg} / \mathrm{L}$ concentrations was in time 15.8 and 22.8 (Figure 1). Expression analysis by $t$-test showed $t$ value $=1.88$ and $p$ value $=0.04$.

In the case of SE191, a Ser83Tyr mutation in gyrase A has been detected. This mutation presented the $0.5 \mathrm{mg} / \mathrm{L} \mathrm{MIC}$ value. In SE23, amino acid substitutions have not been at $0.5 \mathrm{mg} / \mathrm{L}$ exposition.

MLST has been conducted on both strains. K. pneumoniae SE191 was identified as ST274, while K. pneumoniae SE23 presented the novel ST2567. This novel sequence type incorporated a new tonB variant 371 , which had two mutations at $\mathrm{C} 48 \mathrm{~A}$ and $\mathrm{G} 61 \mathrm{C}$ compared to its closest match allele 87. Other alleles of K. pneumoniae SE23 yielded gapA
4, infB 31, mdh 13, pgi 1, phoE 1, and rpoB 1. The nucleic acid sequence of novel ton $B$ allele 371 and the MLST profile of ST2567 have been submitted to $K$. pneumoniae database (http://bigsdb.pasteur.fr/klebsiella/klebsiella.html).

\section{Discussion}

Our study demonstrated role of OqxAB efflux pump in $K$. pneumoniae during exposure to increased ciprofloxacin concentrations. $K$. pneumoniae SE23 and K. pneumoniae SE191 belonging to different sequence types ST2567 and ST274, respectively, have been investigated. They showed varying ability to adapt to ciprofloxacin exposition, though both strains carried oq $x A B$ efflux pump. The two tested strains exhibited different ciprofloxacin MIC values 0.06 and $0.5 \mathrm{mg} / \mathrm{L}$, respectively, and both were susceptible based on the EUCAST 2016 documents (valid at the time when this experiment was started). The initial exposure selected resistant strains only in the ST274 clone and it was further exposed to higher ciprofloxacin concentrations untill $8 \mathrm{mg} / \mathrm{L}$. This strain harboured already a Ser83Tyr amino acid substitution in gyrase A subunit, but no other mutations occurred as consequence to ciprofloxacin exposure. An 
upregulation of $\operatorname{oq}_{x} B$ has been detected with a fold change of 22.8 times increase in expression during the ciprofloxacin exposure from 0.5 till $8 \mathrm{mg} / \mathrm{L}$. This upregulation and selection of resistant strains seem clonally dependent as $K$. pneumoniae SE23, a strain of ST2567, could not increase the expression level of oq $x A B$ and failed to adapt to an increasing ciprofloxacin concentration. This upregulation of efflux pump can contribute to the fitness of ST274, as it has been identified as one of the disseminated clones in newborn and adult hospital wards in Hungary [17].

Results of our study are in accordance with recent ciprofloxacin breakpoint modification of EUCAST. Our study had begun in 2016; therefore, antimicrobial susceptibility testing and interpretation of results were based on EUCAST 2016 documents where $0.5 \mathrm{mg} / \mathrm{L}$ ciprofloxacin value was in susceptible range. The resistance breakpoint of Enterobacteriaceae has been revised in 2017 and set $0.5 \mathrm{mg} / \mathrm{L}$ in nonsusceptible range. $K$. pneumoniae SE191 exhibiting $0.5 \mathrm{mg} / \mathrm{L}$ ciprofloxacin MIC was capable of developing further fluoroquinolone resistance by upregulation of $o q x A B$ efflux pump due to ciprofloxacin exposure. By contrast, $K$. pneumoniae SE23 exhibiting $0.06 \mathrm{mg} / \mathrm{L}$ ciprofloxacin MIC and lacking increased oq $x A B$ expression during exposure to ciprofloxacin was not able to adapt to fluoroquinolone exposition and could not develop resistance.

In our study, a novel $K$. pneumoniae sequence type has been detected, namely, ST2567 that was distinguished by two mutations in tonB allele from its closest sequence type 2387. The new K. pneumoniae ST profile has been deposited to MLST database (http://bigsdb.pasteur.fr/klebsiella/klebsiella.html).

Earlier studies demonstrated OqxAB efflux pump in extended-spectrum beta-lactamase-producing and fluoroquinolone-resistant $K$. pneumoniae high-risk clones [19-21]. In Hungary, K. pneumoniae with oq $x A B$ resistance determinant was reported in bloodstream infections [22]. In all these reported studies, tested strains were resistant to fluoroquinolones. In our study, we demonstrated the role of oq $x A B$ efflux pump in $K$. pneumoniae in selection of resistance to fluoroquinolones. Our two tested strains were susceptible to fluoroquinolones, but after exposure to ciprofloxacin, the one with gyrA mutation could be selected to higher levels of fluoroquinolone resistance. Additional chromosomal mutations of gyrA and parC genes in tested strain did not occur, suggesting that the development of resistance during exposure to $0.5-8 \mathrm{mg} / \mathrm{L}$ ciprofloxacin was mainly caused by the increased expression of the oq $x A B$ efflux pump gene. Strains were exposed to the maximum of $8 \mathrm{mg} / \mathrm{L}$ ciprofloxacin concentration, and in human tissues, ciprofloxacin is unable to reach higher concentrations during a per os dosing; thus, the concentrations used in our study can represent a fluoroquinolone exposure during per os therapy.

\section{Data Availability}

Novel K. pneumoniae MLST profile of ST2567 and new tonB variant 371 sequence data have been submitted to MLST database (http://bigsdb.pasteur.fr/2

\section{Conflicts of Interest}

The authors declare that there are no conflicts of interest.

\section{Acknowledgments}

We thank the team of curators of the Institut Pasteur MLST and whole-genome MLST databases for curating the data of K. pneumoniae ST2567 and making them publicly available at http://bigsdb.pasteur.fr/. This study has been financially supported by OTKA Hungarian Research Fund, Grant Number 108481.

\section{References}

[1] P. Nordmann, G. Cuzon, and T. Naas, "The real threat of Klebsiella pneumoniae carbapenemase-producing bacteria," The Lancet Infectious Diseases, vol. 9, no. 4, pp. 228-236, 2009.

[2] C. Mammina, C. Bonura, F. Di Bernardo et al., "Ongoing spread of colistin-resistant Klebsiella pneumoniae in different wards of an acute general hospital, Italy, June to December 2011," Euro Surveillance, vol. 17, no. 33, pii: 20248, 2012.

[3] K. K. Kumarasamy, M. A. Toleman, T. R. Walsh et al., "Emergence of a new antibiotic resistance mechanism in India, Pakistan, and the UK: a molecular, biological, and epidemiological study," The Lancet Infectious Diseases, vol. 10, no. 9, pp. 597-602, 2010.

[4] J. M. Rodríguez-Martínez, J. Machuca, M. E. Cano, J. Calvo, L. Martínez-Martínez, and A. Pascual, "Plasmid-mediated quinolone resistance: two decades on," Drug Resistance Updates, vol. 29, pp. 13-29, 2016.

[5] L. H. Hansen, E. Johannesen, M. Burmølle, A. H. Sørensen, and S. J. Sørensen, "Plasmid-encoded multidrug efflux pump conferring resistance to olaquindox in Escherichia coli," Antimicrobial Agents and Chemotherapy, vol. 48, no. 9, pp. 3332-3337, 2004.

[6] L. H. Hansen, L. B. Jensen, H. I. Sørensen, and S. J. Sørensen, "Substrate specificity of the OqxAB multidrug resistance pump in Escherichia coli and selected enteric bacteria," Journal of Antimicrobial Chemotherapy, vol. 60, no. 1, pp. 145-147, 2007.

[7] J.M. Rodríguez-Martínez, P. Díaz de Alba, A. Briales et al., "Contribution of OqxAB efflux pumps to quinolone resistance in extended-spectrum- $\beta$-lactamase-producing Klebsiella pneumoniae," Journal of Antimicrobial Chemotherapy, vol. 68, no. 1, pp. 68-73, 2013.

[8] F. Perez, S. D. Rudin, S. H. Marshall et al., "OqxAB, a quinolone and olaquindox efflux pump, is widely distributed among multidrug-resistant Klebsiella pneumoniae isolates of human origin," Antimicrobial Agents and Chemotherapy, vol. 57, no. 9, pp. 4602-4603, 2013.

[9] H. B. Kim, C. H. Park, C. J. Kim, E. C. Kim, G. A Jacoby, and D. C. Hooper, "Prevalence of plasmid-mediated quinolone resistance determinants over a 9-year period," Antimicrobial Agents and Chemotherapy, vol. 53, no. 2, pp. 639-645, 2009.

[10] H. B. Kim, M. Wang, C. H. Park, E. C. Kim, G. A. Jacoby, and D. C. Hooper, "oq $x A B$ encoding a multidrug efflux pump in human clinical isolates of Enterobacteriaceae," Antimicrobial Agents and Chemotherapy, vol. 53, no. 8, pp. 3582-3584, 2009.

[11] S. De Majumdar, M. Veleba, S. Finn, S. Fanning, and T. Schneiders, "Elucidating the regulon of multidrug resistance regulator RarA in Klebsiella pneumoniae," Antimicrobial Agents and Chemotherapy, vol. 57, no. 4, pp. 1603-1609, 2013.

[12] M. Veleba, P. G. Higgins, G. Gonzalez, H. Seifert, and T. Schneiders, "Characterization of RarA, a novel AraC family multidrug resistance regulator in Klebsiella pneumoniae," 
Antimicrobial Agents and Chemotherapy, vol. 56, no. 8, pp. 4450-4458, 2012.

[13] X. Zhong, H. Xu, D. Chen, H. Zhou, X. Hu, and G. Cheng, "First emergence of $a c r A B$ and oqxAB mediated tigecycline resistance in clinical isolates of Klebsiella pneumoniae predating the use of tigecycline in a Chinese hospital," PLoS One, vol. 9, no. 12, Article ID e115185, 2014.

[14] S. Bialek-Davenet, J.P. Lavigne, K. Guyot et al., "Differential contribution of $\mathrm{Acr} A \mathrm{~B}$ and OqxAB efflux pumps to multidrug resistance and virulence in Klebsiella pneumoniae," Journal of Antimicrobial Chemotherapy, vol. 70, no. 1, pp. 81-88, 2015.

[15] I. Damjanova, A. Tóth, J. Pászti et al., "Expansion and countrywide dissemination of ST11, ST15 and ST147 ciprofloxacin-resistant CTX-M-15-type beta-lactamaseproducing Klebsiella pneumoniae epidemic clones in Hungary in 2005 the new 'MRSAs'?," Journal of Antimicrobial Chemotherapy, vol. 62, no. 5, pp. 978-985, 2008.

[16] I. Damjanova, A. Tóth, J. Pászti, A. Bauernfeind, and M. Füzi, "Nationwide spread of clonally related CTX-M-15-producing multidrug-resistant Klebsiella pneumoniae strains in Hungary," European Journal of Clinical Microbiology \& Infectious Diseases, vol. 25, pp. 275-278, 2006.

[17] A. Tóth, B. Kocsis, I. Damjanova et al., "Fitness cost associated with resistance to fluoroquinolones is diverse across clones of Klebsiella pneumoniae and may select for CTX-M-15 type extended-spectrum $\beta$-lactamase," European Journal of Clinical Microbiology \& Infectious Diseases, vol. 33, no. 5, pp. 837-843, 2014.

[18] L. Diancourt, V. Passet, J. Verhoef, P. A. Grimont, and S. Brisse, "Multilocus sequence typing of Klebsiella pneumoniae nosocomial isolates," Journal of Clinical Microbiology, vol. 43, no. 8, pp. 4178-4182, 2005.

[19] L. Cheng, X. L. Cao, Z. F. Zhang et al., "Clonal dissemination of KPC-2 producing Klebsiella pneumoniae ST11 clone with high prevalence of $o q x A B$ and $r m t B$ in a tertiary hospital in China: results from a 3-year period," Annals of Clinical Microbiology and Antimicrobials, vol. 15, no. 1, pp. 1-8, 2016.

[20] J. Anes, D. Hurley, M. Martins, and S. Fanning, "Exploring the genome and phenotype of multi-drug resistant Klebsiella pneumoniae of clinical origin," Frontiers in Microbiology, vol. 8, p. 1913, 2017.

[21] A. Abderrahim, N. Djahmi, C. Pujol et al., "First case of NDM-1-producing Klebsiella pneumoniae in Annaba University Hospital, Algeria," Microbial Drug Resistance, vol. 23, no. 7, pp. 895-900, 2017.

[22] J. Domokos, K. Kristóf, and D. Szabó, "Plasmid-mediated quinolone resistance among extended-spectrum betalactamase producing Enterobacteriaceae from bloodstream infections," Acta Microbiologica et Immunologica Hungarica, vol. 63, no. 3, pp. 313-323, 2016. 


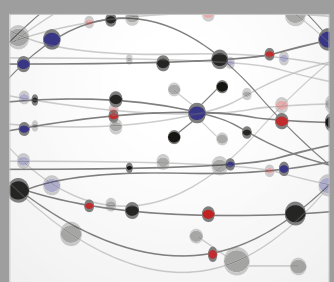

The Scientific World Journal
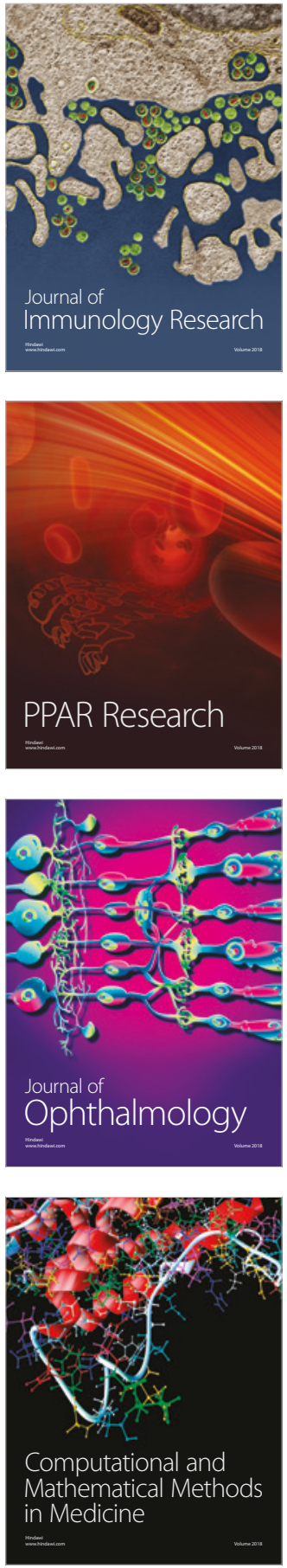

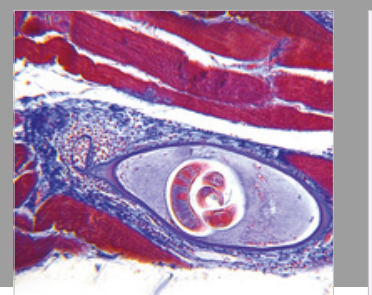

Gastroenterology Research and Practice

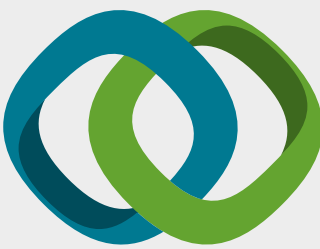

\section{Hindawi}

Submit your manuscripts at

www.hindawi.com
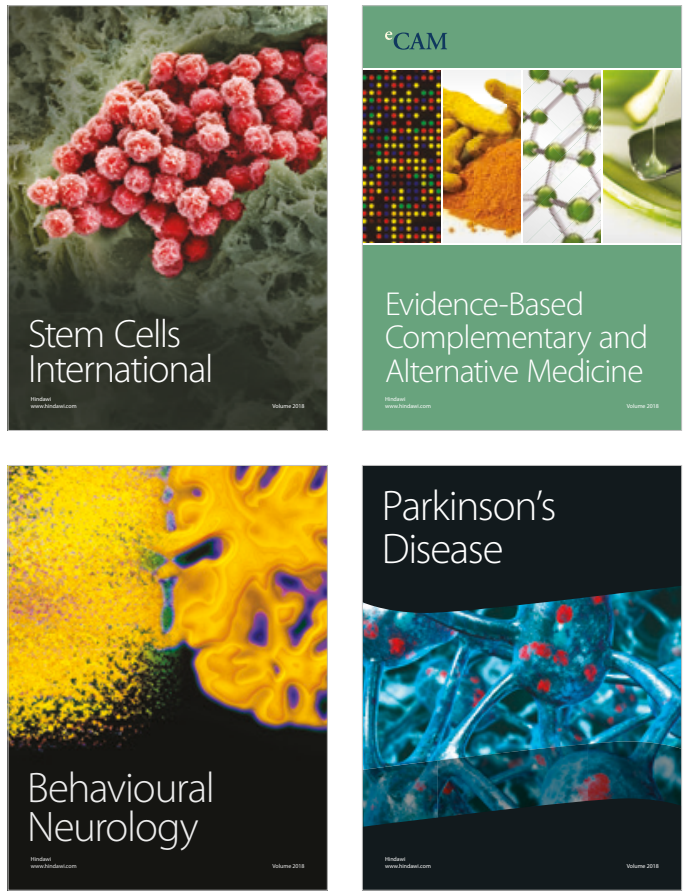

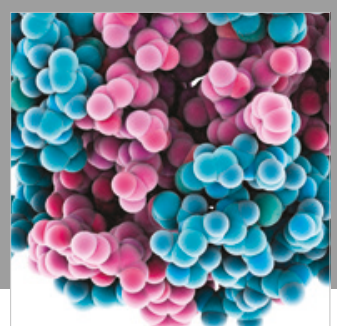

ournal of

Diabetes Research

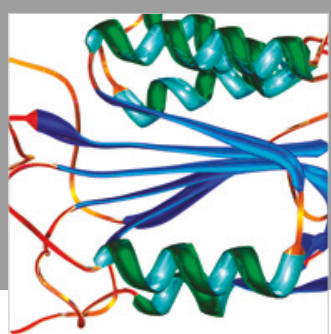

Disease Markers
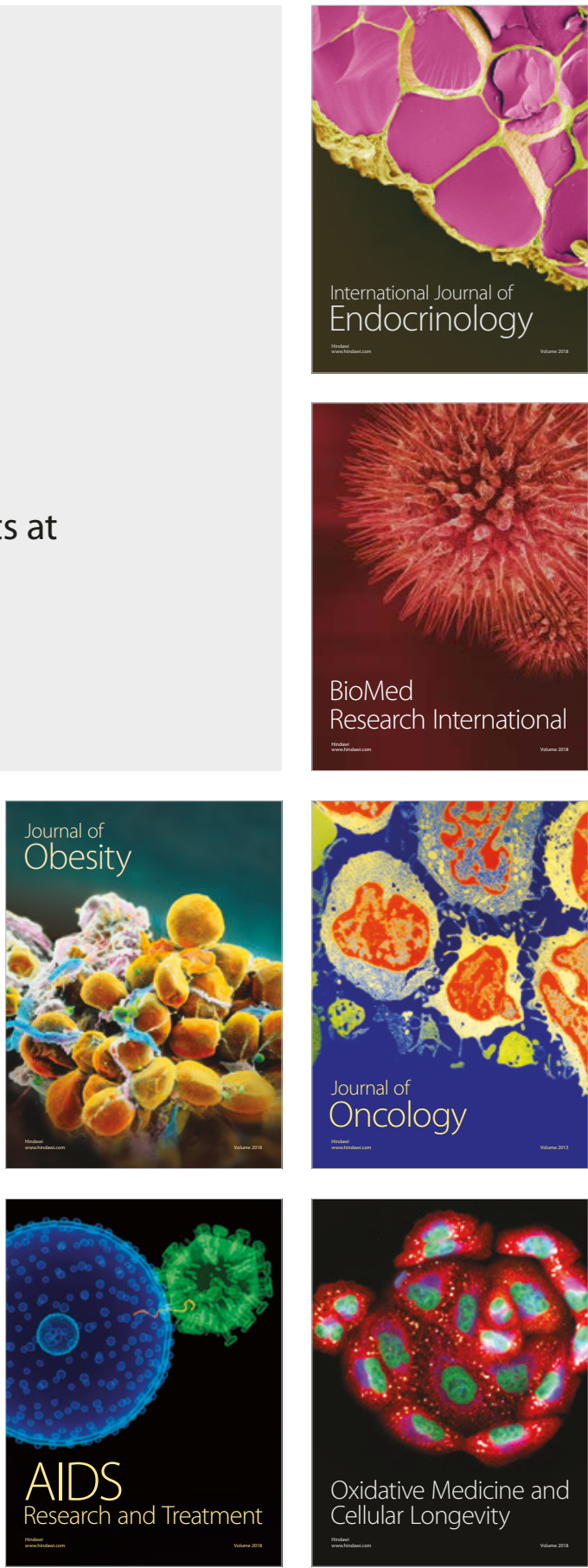